ISSN 0103-5150

Fisioter. Mov., Curitiba, v. 25, n. 1, p. 219-230, jan./mar. 2012

Licenciado sob uma Licença Creative Commons

\title{
Teste de força/resistência de membros superiores: análise metodológica e dados normativos
}

\author{
Strength/resistance test of the superior members: \\ methodological analysis and normative data
}

\section{Bruno Ferreira Marinho ${ }^{[a]}$, João Carlos Bouzas Marins ${ }^{[b]}$}

[a] Aluno não vinculado ao Programa de Pós-Graduação Stricto Sensu em nível de Mestrado da Universidade Federal de Viçosa (UFV), Viçosa, MG - Brasil, e-mail: bruno.marinho@rocketmail.com

[b] Professor Doutor do Departamento de Educação Física da Universidade Federal de Viçosa (UFV), Viçosa, MG - Brasil, e-mail: jcbouzas@ufv.br

\section{Resumo}

Introdução: A força e a resistência muscular são componentes da aptidão física importantes para a saúde e alto rendimento. Os testes de flexão de braços, puxada em suspensão na barra e puxada em suspensão na barra modificada, utilizam o próprio peso corporal e avaliam as capacidades motoras relacionadas à aptidão física, saúde e ao desempenho atlético, no sentido de mensurar a força e resistência muscular dos membros superiores em ambos os sexos e ampla faixa etária. Objetivos: 0 objetivo deste estudo foi buscar na literatura os aspectos metodológicos relacionados à validade, fidedignidade, objetividade e procedimentos específicos utilizados nos testes: flexão de braços, puxada suspensão na barra e puxada em suspensão na barra modificada, fazendo, dessa forma, um levantamento dos indicadores de rendimento desses testes em atletas de alto nível de diferentes modalidades. Conclusão: Os testes de flexão de braços, puxada em suspensão na barra e puxada em suspensão na barra modificada são eficientes, confiáveis, de fácil aplicação e são ferramentas que empregam baixo custo, além de requererem pouco treinamento do avaliador. Entretanto, devemos chamar a atenção quanto à padronização e as recomendações metodológicas para suas aplicações, para que possam ser utilizados com segurança na reabilitação e no desempenho atlético.

Palavras-chave: Avaliação física. Força. Flexão de braços. Puxada em suspensão na barra. Puxada em suspensão na barra modificada. 


\begin{abstract}
Introduction: The strength and muscular endurance are important components of physical fitness for health and high performance. The tests of push-up, pull-up and modified pull-up, using own body weight and evaluate motor skills related to physical fitness, health and athletic performance, to measure the strength and muscular endurance upper limbs in both sexes and a wide age range. Objectives: The aim of this study was to search the literature methodological issues related to validity, reliability, objectivity and specific procedures used in tests: push-up, pull-up and modified pull-up, by doing so, a survey of indicators yield of these tests in elite athletes of different modalities. Conclusion: The tests of push-up, pull-up and modified pull-up are efficient, reliable, easy to apply and are tools that employ low cost, and require little training for evaluators. However, as we draw attention to the standardization and methodological recommendations for your applications so they can be safely used in rehabilitation and athletic performance.
\end{abstract}

Keywords: Physical assessment. Strength. Push-up. Pull-up. Modified pull-up.

\section{Introdução}

0 conceito da aptidão física foi delineado por meio do modelo multidimensional a partir da década de 1960, sendo relacionado à saúde que avalia o componente cardiovascular e neuromuscular (1). Tendo em vista que o sedentarismo, ou má condição física, está associado ao desenvolvimento de doenças crônicas e morte prematura (2), a força e a resistência muscular são componentes da aptidão física importantes, sendo necessários níveis mínimos para realização de atividades diárias (3), como, por exemplo, carregar, apoiar e tracionar objetos pesados. Morrow (4) considera importante avaliar a força pelo fato de ser essencial tanto para a aptidão física relacionada à saúde quanto para o desempenho atlético, auxiliando também na identificação de talentos esportivos.

Os testes que utilizam o próprio peso corporal, a fim de verificar a força e a resistência muscular, são muito utilizados por apresentarem baixo custo e utilizarem equipamentos acessíveis (5). Dentre os testes motores que verificam a força/resistência dinâmica dos membros superiores, os utilizados mais frequentemente são: flexão de braços, puxada em suspensão na barra e puxada em suspensão na barra modificada (6). Tendo em vista que a massa corporal afeta a validade dos testes, foram propostas modificações no teste de puxada em suspensão na barra (7), permitindo o apoio dos pés sobre o solo, minimizando a sobrecarga excessiva do peso corporal. A partir dessas modificações, surgiu o teste puxada em suspensão na barra modificada.
Com uma pesquisa realizada na base de dados PubMed, foram reportados os estudos de Eckert e Day (8), Cotten (9) e Baumgartner (10) como os primeiros estudos utilizando os testes de flexões de braços, puxada em suspensão na barra e puxada em suspensão na barra modificada. Entretanto, o texto completo dos artigos, incluindo os resumos, não está disponível pela base de dados consultada.

$\mathrm{Na}$ literatura referente a lutas e artes marciais, como luta olímpica (11-13), taekwondo (14) e jiu-jitsu (15), esses testes são muitos utilizados, talvez pelo baixo custo em relação a outros testes mais dispendiosos, e também pela facilidade de aplicação, podendo ser aplicados em vários indivíduos ao mesmo tempo, em um pequeno espaço de tempo.

Cabe salientar a importância da utilização de testes motores na avaliação, pois oferecem indicativos sobre o estado de condicionamento físico de um atleta e, a partir disso, podem ser feitas intervenções em relação ao treinamento. Tendo em vista que esses testes possuem diferenças metodológicas na aplicação, entre os gêneros e diferentes populações (2), a padronização dos procedimentos metodológicos utilizados nesses testes visa a auxiliar treinadores, professores e técnicos quanto à execução, padronização e possíveis erros durante a aplicação desses testes.

Por fim, objetivou-se fazer uma revisão das ações metodológicas específicas utilizadas nos testes: flexão de braços, puxada suspensão na barra e puxada em suspensão na barra modificada em atletas de alto nível. Realizou-se, dessa forma, um levantamento dos indicadores de rendimento desses testes em atletas de alto nível de diferentes modalidades. 


\section{Metodologia}

Foi realizada uma pesquisa nas bases de dados PubMed, EBSCO e SciELO, utilizando associações das palavras-chave: pull-up, push-up, modified pull-up, fitness performance, fitness profile, pull-ups, push-up. Com base nesta pesquisa, foram selecionados, a partir da leitura de seus resumos, 26 estudos que avaliam o desempenho muscular de membros superiores, nos idiomas inglês, português e espanhol. Além disso, foi realizada exaustiva busca em livros especializados. Em seguida, buscou-se identificar os aspectos metodológicos relacionados à validade, fidedignidade, objetividade e procedimentos, visando a estabelecer dados normativos em atletas de alto rendimento em suas respectivas modalidades e em pessoas saudáveis.

\section{Qualidades psicométricas}

A validade, a fidedignidade e a objetividade na avaliação são qualidades psicométricas, representando essas qualidades a especialização em testes e avaliações (16). A validade se refere à veracidade de um instrumento de avaliação, permitindo que sejam feitas inferências significativas a partir dos resultados. A fidedignidade nos fornece a confiança de que o resultado de um examinado seria aproximadamente o mesmo caso o mesmo teste ou avaliação fosse reproduzido novamente. Já a objetividade de um teste refere-se à exatidão dos resultados de um determinado instrumento de avaliação, para que possa ser utilizado por outros examinadores, a fim de obter resultados semelhantes (16) (Quadros 1 e 2).

\section{Procedimentos metodológicos dos testes}

\section{Teste de flexão de braços}

Recomendações metodológicas para sua aplicação: o avaliado deve se posicionar em decúbito ventral, com as mãos apoiadas no solo, com uma distância de 10 a $20 \mathrm{~cm}$ a partir da linha dos ombros, com os dedos voltados para frente (19). 0 posicionamento das mãos sobre o solo não deve ser acima da linha dos ombros e, na posição inicial do movimento, o rosto deve permitir um alinhamento adequado entre o tronco e as pernas (20) (Figuras
Quadro 1 - Flexão de braços - push-up

\begin{tabular}{|c|c|}
\hline Gênero & Masculino \\
\hline $\begin{array}{l}\text { Objetivo do } \\
\text { teste }\end{array}$ & $\begin{array}{l}\text { Avaliar a força/resistência dos músculos dos } \\
\text { membros superiores e da cintura escapular no } \\
\text { movimento de flexão e extensão dos cotovelos } \\
\text { sobre o solo (6) }\end{array}$ \\
\hline Validade & $\begin{array}{l}\text { Foi reportado um "r" de } 0,93(17) \text {, podendo ter } \\
\text { sua validade aumentada quando corrigida pela } \\
\text { massa corporal (2) }\end{array}$ \\
\hline Fidedignidade & Apresenta valores elevados, "r" 0.93 (4) \\
\hline Objetividade & Foi obtido um "r" de 0.99 (18) \\
\hline
\end{tabular}

Fonte: Dados da pesquisa.

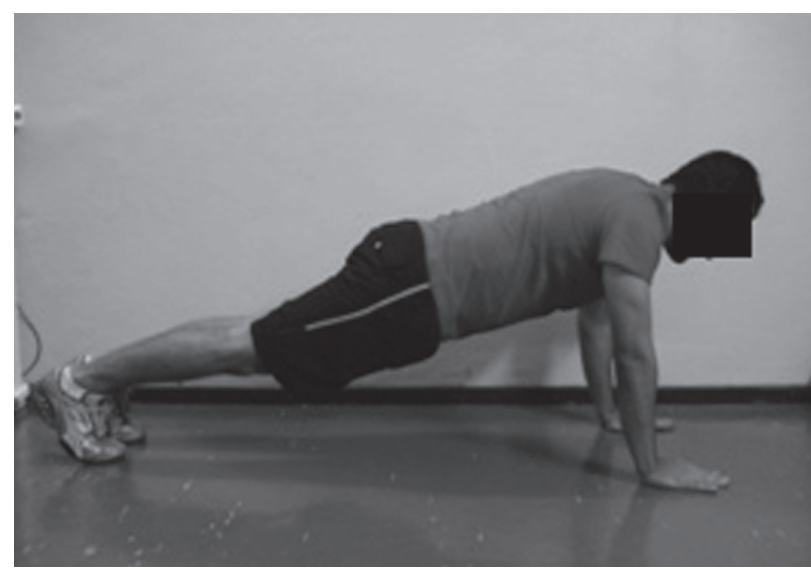

Figura 1 - Posição inicial

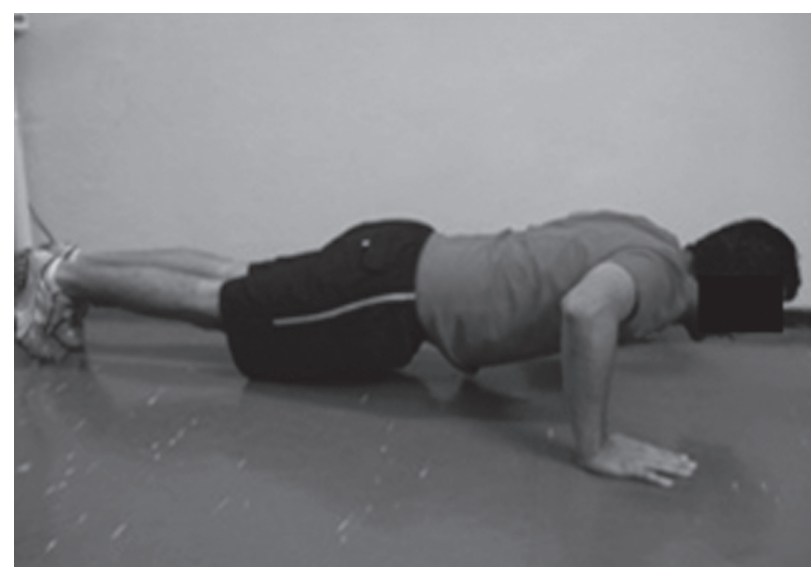

Figura 2 - Posição final

1, 2). A aplicação do teste para o sexo feminino é modificada apenas pelo apoio dos joelhos sobre o solo (18). Os demais procedimentos são realizados para ambos os sexos. É recomendado que o avaliado execute algumas vezes o movimento para melhor 
aprendizagem do teste, porém essas realizações prévias podem causar desgaste muscular, podendo interferir nos resultados ou prejudicar uma futura comparação entre os dados obtidos (21).

Resultado: registra-se o número máximo de repetições corretas em um minuto (22).

Indicação metodológica para os desportos: pode ser aplicado em diversos desportos, como esgrima, basquetebol, handebol, voleibol, karatê, judô, jiu-jitsu, luta olímpica, ginástica olímpica, ginástica rítmica (23), entre outros, em que a força dinâmica de membros superiores seja de grande importância.

Possíveis erros de aplicação: 0 teste deve ser interrompido se o avaliado realizar uma execução incorreta ou não completar o movimento de flexão e extensão de cotovelos. Cabe ressaltar que a carga levantada é relativa à massa corporal do indivíduo. Sendo assim, a gordura corporal e o comprimento do tronco e dos membros podem influenciar nos resultados (4). Por exigir força e resistência dos membros superiores, o teste exige uma habilidade mínima para que seja realizado (17).

Equipamentos: pode ser utilizado com um colchonete no chão (18).

Os valores normativos do teste de flexão de braços podem ser vistos na sequência (Tabela 1 ).

\section{Puxada em suspensão de barra}

Recomendações metodológicas para sua aplicação: nos homens, o avaliado deve se posicionar em suspensão vertical, com extensão total dos braços e pernas sem que haja contato dos pés com o solo. As mãos devem estar fixas na barra com empunhadura pronada (dorso das mãos voltado para o indivíduo), correspondendo à largura dos ombros. Partindo dessa posição, o indivíduo eleva seu corpo até que o seu queixo ultrapasse o nível da barra, e volta à posição inicial (Figuras 3, 4). Para o sexo feminino, esse teste é diferenciado pelo posicionamento estático, sendo o resultado o registro do tempo máximo em segundos de permanência em suspensão com o queixo acima do nível da barra. Esse teste também é conhecido como suspensão na barra - flexed arm hang $(18,24)$.

Resultado: para o sexo masculino, registra-se o número máximo de repetições corretas $(7,18)$. Para o sexo feminino, registra-se o tempo máximo em segundos, em permanência com o queixo acima do nível da barra.

Indicação metodológica para os desportos: para ambos os sexos, a literatura demonstra grande aplicação desse teste nas modalidades de lutas e artes marciais $(11-12,25)$, e também no militarismo (26-27).

Possíveis erros de aplicação: para o sexo masculino, deve-se evitar a realização do teste com ajuda do balanço do corpo, e que em cada movimento o queixo ultrapasse o nível da barra $(18,24)$. Os movimentos devem ser contínuos, não permitindo descanso entre uma repetição e outra (7). Cabe ao avaliador orientar verbalmente ou demonstrando o movimento antes do indivíduo começar o teste, para que as repetições prévias realizadas para melhor aprendizagem do teste não interfiram em seu

Tabela 1 - Valores normativos do teste de flexão de braços em pessoas saudáveis

\begin{tabular}{|c|c|c|c|c|c|c|c|c|c|c|c|c|}
\hline \multirow{2}{*}{$\begin{array}{l}\text { Faixa etária } \\
\text { Gênero }\end{array}$} & \multicolumn{2}{|c|}{$15-19$} & \multicolumn{2}{|c|}{$20-29$} & \multicolumn{2}{|c|}{$30-39$} & \multicolumn{2}{|c|}{$40-49$} & \multicolumn{2}{|c|}{ 50-59 } & \multicolumn{2}{|c|}{$60-69$} \\
\hline & $M$ & $\mathrm{~F}$ & $M$ & $\mathrm{~F}$ & $M$ & $\mathrm{~F}$ & $M$ & $\mathrm{~F}$ & $M$ & $\mathrm{~F}$ & $M$ & $\mathrm{~F}$ \\
\hline Excelente & $\geq 39$ & $\geq 33$ & $\geq 36$ & $\geq 30$ & $\geq 30$ & $\geq 27$ & $\geq 22$ & $\geq 24$ & $\geq 21$ & $\geq 21$ & $\geq 18$ & $\geq 17$ \\
\hline $\begin{array}{l}\text { Acima da } \\
\text { média }\end{array}$ & $29-38$ & $25-32$ & $29-35$ & $21-29$ & $22-29$ & $20-26$ & $17-21$ & $15-23$ & $13-20$ & $11-20$ & $11-17$ & $12-16$ \\
\hline Na média & $23-28$ & $18-24$ & $22-28$ & $15-20$ & $17-21$ & $13-19$ & $13-16$ & $11-14$ & $10-12$ & $07-10$ & $08-10$ & $05-11$ \\
\hline $\begin{array}{l}\text { Abaixo da } \\
\text { média }\end{array}$ & $18-22$ & $12-17$ & $17-21$ & $10-14$ & $12-16$ & $08-12$ & $10-12$ & $05-10$ & 07-09 & $02-06$ & $05-07$ & $02-04$ \\
\hline Ruim & $\leq 17$ & $\leq 11$ & $\leq 16$ & $\leq 09$ & $\leq 11$ & $\leq 07$ & $\leq 09$ & $\leq 04$ & $\leq 06$ & $\leq 01$ & $\leq 04$ & $\leq 01$ \\
\hline
\end{tabular}

Fonte: Pollock (22). 
Quadro 2 - Puxada em suspensão na barra - pull-up

\begin{tabular}{|c|c|}
\hline Gênero & Feminino \\
\hline $\begin{array}{l}\text { Objetivo do } \\
\text { teste }\end{array}$ & $\begin{array}{l}\text { Avaliar a força/resistência dos músculos dos } \\
\text { membros superiores e da cintura escapular } \\
\text { com o corpo em suspensão em uma barra fixa } \\
\text { por meio do movimento de flexão e extensão } \\
\text { dos cotovelos (6) }\end{array}$ \\
\hline Validade & Valor não reportado (18) \\
\hline Fidedignidade & Um "r" de 0.87 (18) Um "r" de 0.90 (18) \\
\hline Objetividade & Foi reportado um "r" de 0.99 (18) \\
\hline
\end{tabular}

Fonte: Dados da pesquisa.

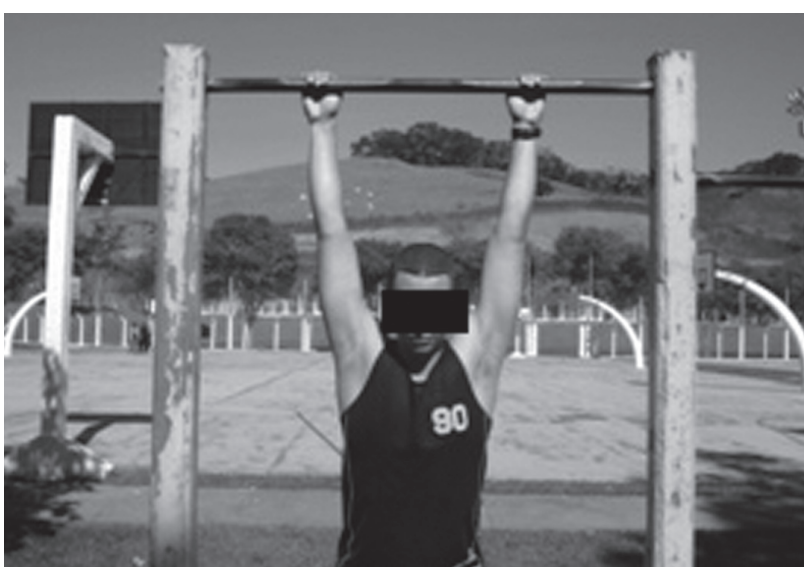

Figura 3 - Posição inicial

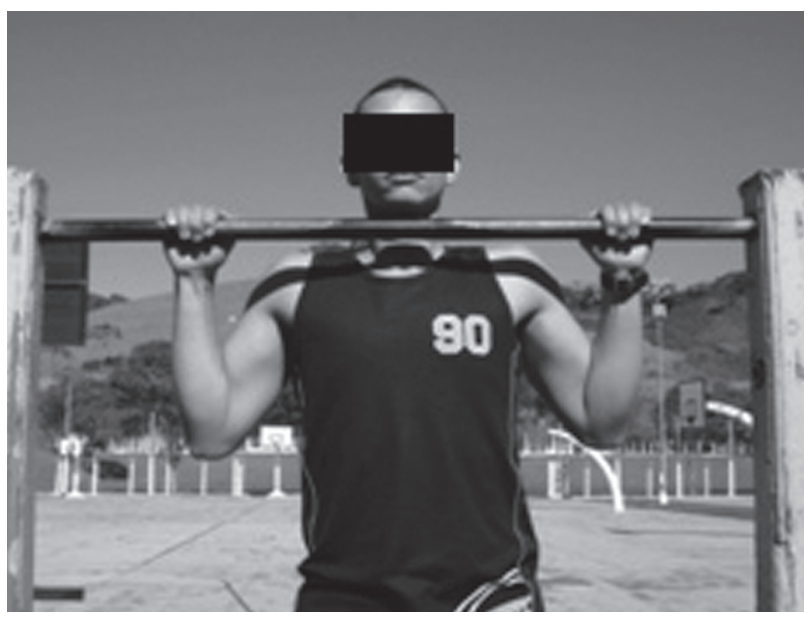

Figura 4 - Posição final

resultado, por conta da fadiga muscular (6). Para o sexo feminino, deve-se ter o cuidado de acionar o cronômetro assim que a avaliada assuma a posição em suspensão na barra e de travá-lo quando o queixo da avaliada tocar a barra ou estiver abaixo do nível da barra ou, ainda, quando a avaliada colocar a cabeça para trás para não tocar a barra $(18,24)$.

Equipamentos: utiliza-se uma barra de metal na horizontal, medindo aproximadamente um metro e meio de comprimento, com três centímetros de diâmetro. A altura da barra deve permitir que o avaliado fique em suspensão na barra sem o contato dos pés com o solo (18).

Na sequência, podem ser vistos os valores de percentil encontrados para o teste de puxada em suspensão na barra (Tabela 2).

Puxada em suspensão na barra modificada modified pull-up

0 teste de puxada em suspensão na barra tem sua validade discutida, pela interferência da massa corporal, afetando negativamente os resultados obtidos $(7,28)$. Nesse sentido, foram propostas modificações nesse teste, permitindo que o avaliado apoie os pés sobre o solo. Essa nova versão de teste, puxada em suspensão na barra modificada, minimiza a sobrecarga imposta pela massa corporal, amenizando as exigências de força máxima impostas pelo teste $(6-7,29)$. Entretanto, não foram encontrados dados na literatura que referenciem valores em atletas, sendo a maioria dos estudos voltados para aptidão física de crianças e adolescentes (28-34). Guedes (7) salienta que a relação entre o número de repetições/peso corporal possa aumentar sua validade. No entanto, esse procedimento ainda é considerado objeto de estudo (6-7), com alguns autores sugerindo que sua utilização ainda é prematura $(7,28)$.

A seguir serão descritas todas as orientações quanto aos procedimentos e recomendações metodológicas para sua aplicação:

Objetivo do teste: avaliar a força/resistência dos músculos dos membros superiores e da cintura escapular com o corpo em suspensão em uma barra fixa por meio do movimento de flexão e extensão dos cotovelos (6).

Recomendações metodológicas para sua aplicação: o posicionamento da barra deve estar $3 \mathrm{~cm}$ acima da altura dos dedos, estando o indivíduo em decúbito dorsal, com os braços totalmente estendidos. 0 indivíduo coloca-se pendurado - com cotovelos em extensão -, a barra em direção de seus ombros, o corpo 
Tabela 2 - Valores em percentil para o teste de puxada em suspensão na barra - pull-up, em pessoas saudáveis

\begin{tabular}{|c|c|c|c|c|c|c|c|c|c|c|c|c|c|c|c|c|}
\hline \multicolumn{17}{|c|}{ Idade } \\
\hline \multirow[b]{2}{*}{ Percentil } & \multicolumn{2}{|c|}{$9-10$} & \multicolumn{2}{|c|}{11} & \multicolumn{2}{|c|}{12} & \multicolumn{2}{|c|}{13} & \multicolumn{2}{|c|}{14} & \multicolumn{2}{|c|}{15} & \multicolumn{2}{|c|}{16} & \multicolumn{2}{|c|}{$17+$} \\
\hline & M & $F$ & M & $\mathrm{F}$ & M & $\mathrm{F}$ & M & $F$ & M & $F$ & M & $\mathrm{F}$ & $M$ & $\mathrm{~F}$ & $M$ & $\mathrm{~F}$ \\
\hline 100 & 19 & 78 & 16 & 68 & 18 & 84 & 17 & 68 & 27 & 65 & 20 & 83 & 26 & 69 & 23 & 73 \\
\hline 95 & 9 & 42 & 8 & 39 & 9 & 33 & 10 & 34 & 12 & 35 & 15 & 36 & 14 & 31 & 15 & 34 \\
\hline 90 & 7 & 29 & 6 & 30 & 7 & 27 & 9 & 25 & 10 & 29 & 12 & 28 & 12 & 24 & 13 & 28 \\
\hline 85 & 5 & 24 & 5 & 24 & 6 & 23 & 7 & 21 & 9 & 26 & 11 & 25 & 11 & 20 & 12 & 22 \\
\hline 80 & 4 & 21 & 5 & 21 & 5 & 21 & 6 & 20 & 8 & 23 & 10 & 21 & 10 & 17 & 11 & 19 \\
\hline 75 & 3 & 18 & 4 & 20 & 4 & 18 & 5 & 16 & 7 & 21 & 9 & 18 & 10 & 15 & 10 & 17 \\
\hline 70 & 3 & 16 & 4 & 17 & 4 & 15 & 5 & 14 & 7 & 18 & 9 & 15 & 9 & 12 & 10 & 14 \\
\hline 65 & 2 & 14 & 3 & 15 & 3 & 13 & 4 & 13 & 6 & 15 & 8 & 14 & 8 & 11 & 9 & 12 \\
\hline 60 & 2 & 12 & 3 & 13 & 3 & 12 & 4 & 11 & 5 & 13 & 7 & 12 & 8 & 10 & 8 & 10 \\
\hline 55 & 1 & 10 & 2 & 11 & 2 & 10 & 3 & 9 & 5 & 11 & 7 & 10 & 7 & 8 & 7 & 9 \\
\hline 50 & 1 & 9 & 2 & 10 & 2 & 9 & 3 & 8 & 4 & 9 & 6 & 9 & 7 & 7 & 7 & 8 \\
\hline 45 & 1 & 7 & 1 & 8 & 1 & 8 & 2 & 7 & 4 & 8 & 5 & 8 & 6 & 6 & 6 & 7 \\
\hline 40 & 1 & 6 & 1 & 7 & 1 & 6 & 2 & 6 & 3 & 7 & 5 & 7 & 6 & 5 & 6 & 6 \\
\hline 35 & 1 & 5 & 1 & 6 & 1 & 5 & 2 & 5 & 3 & 5 & 4 & 5 & 5 & 4 & 5 & 5 \\
\hline 30 & 0 & 4 & 1 & 5 & 0 & 4 & 1 & 4 & 2 & 5 & 4 & 4 & 5 & 3 & 5 & 4 \\
\hline 25 & 0 & 3 & 0 & 3 & 0 & 3 & 1 & 3 & 2 & 3 & 3 & 4 & 4 & 3 & 4 & 3 \\
\hline 20 & 0 & 2 & 0 & 3 & 0 & 2 & 0 & 2 & 1 & 3 & 2 & 3 & 3 & 2 & 3 & 2 \\
\hline 15 & 0 & 1 & 0 & 2 & 0 & 1 & 0 & 1 & 1 & 2 & 1 & 2 & 3 & 1 & 2 & 2 \\
\hline 10 & 0 & 0 & 0 & 0 & 0 & 1 & 0 & 0 & 0 & 1 & 1 & 1 & 2 & 1 & 1 & 1 \\
\hline 5 & 0 & 0 & 0 & 0 & 0 & 0 & 0 & 0 & 0 & 0 & 0 & 0 & 1 & 0 & 0 & 0 \\
\hline 0 & 0 & 0 & 0 & 0 & 0 & 0 & 0 & 0 & 0 & 0 & 0 & 0 & 0 & 0 & 0 & 0 \\
\hline
\end{tabular}

Nota: No masculino é contado o número de repetições do movimento; já no feminino é considerado o tempo em segundos em suspensão na barra.

Fonte: Johnson (18). 
ereto e apenas os calcanhares em contato com solo. A posição das mãos com empunhadura pronada e equivalente à largura dos ombros. Eleva-se o corpo até que a região da garganta toque a linha de demarcação colocada a dois espaços abaixo da barra e então se retorna o corpo à posição inicial, completando uma repetição (Figuras 5, 6). 0 movimento deve ser repetido o máximo de vezes possível, sem limite de tempo, sem paralisações entre as repetições e sem colocar nenhuma parte do corpo em contato com o solo, a não ser os calcanhares $(6,29)$.

Resultado: refere-se ao número máximo de repetições corretas em uma única tentativa $(6,29)$, sem limite de tempo.

Possíveis erros de aplicação: deve-se orientar que o avaliado estenda os braços no início de cada movimento, que a região da garganta deve tocar a linha de demarcação no fim de cada repetição, e que não é permitido realizar nenhum movimento dos quadris ou pernas, a fim de minimizar a sobrecarga dos braços (7).

Equipamentos: uma armação de madeira para fixar a barra, com dimensões de 120 x $50 \mathrm{~cm}$ na base e caibros de $12 \times 8 \mathrm{~cm}$ acoplados à base para suporte da barra de metal circular com aproximadamente $150 \mathrm{~cm}$ de comprimento e $3 \mathrm{~cm}$ de diâmetro. Os caibros que suportam a barra devem ter $140 \mathrm{~cm}$,

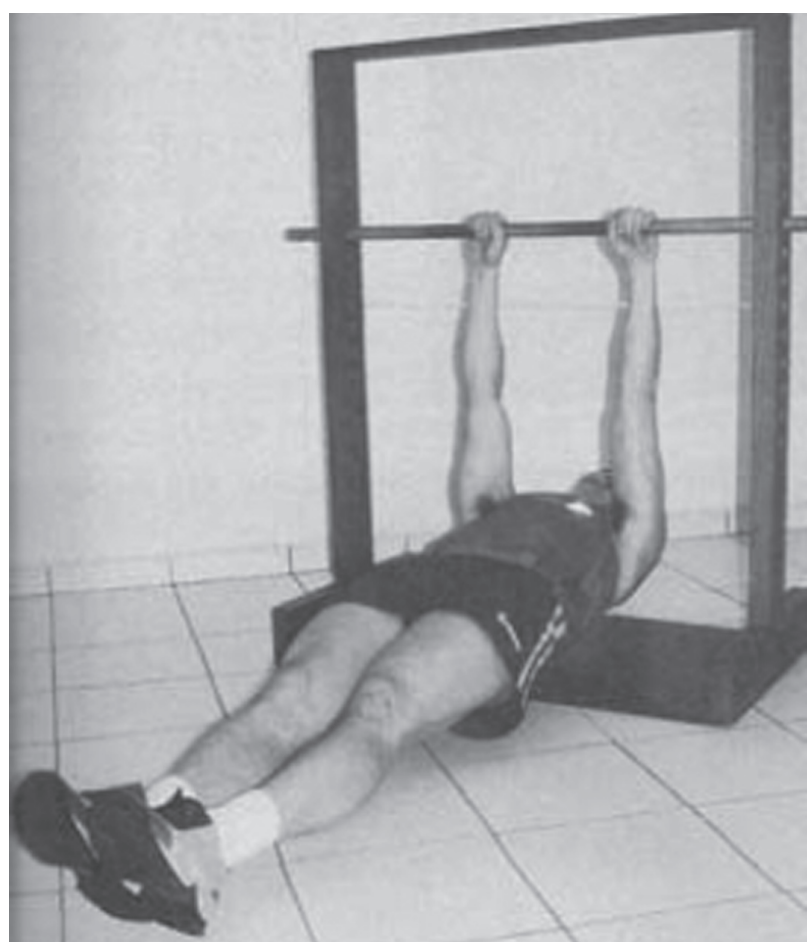

Figura 5 - Posição inicial Fonte: Guedes (6).

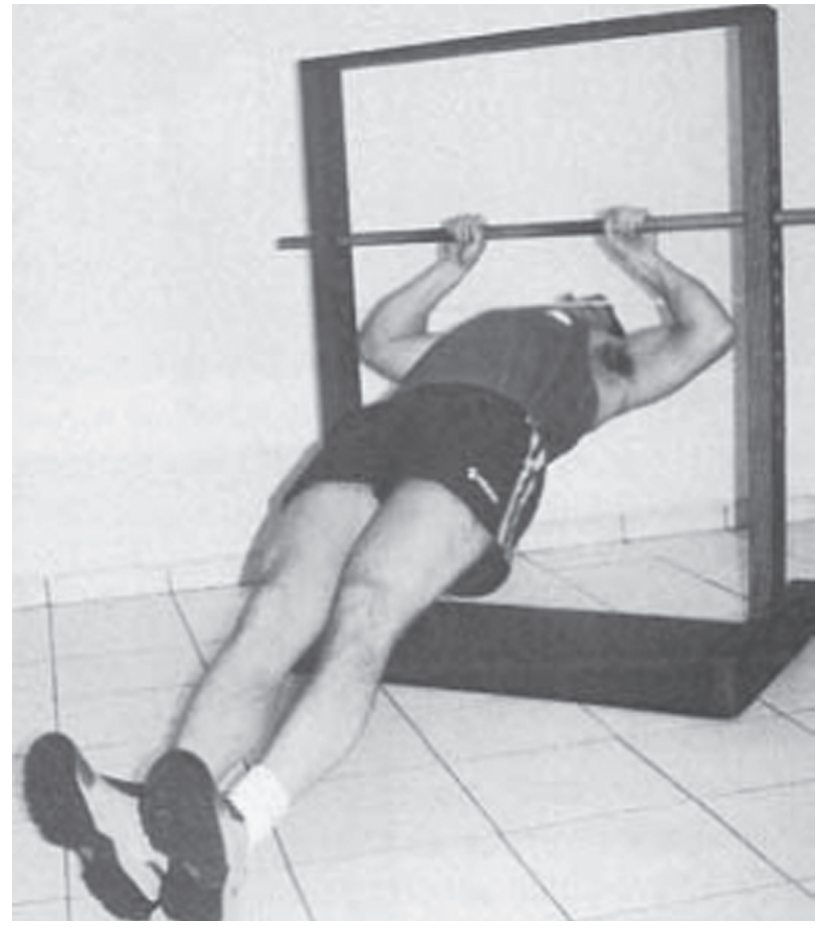

Figura 6 - Posição final

Fonte: Guedes (6).

com orifícios a cada $5 \mathrm{~cm}$, para que a barra possa ser ajustada ao comprimento dos braços. Uma tábua suspensa de $12 \mathrm{~cm}$ de largura por $1,5 \mathrm{~cm}$ de espessura fixada acima dos caibros de suporte evita que a armação possa movimentar-se durante a realização dos movimentos $(6,29)$.

Na sequência podem ser vistos os valores em percentil para o teste de puxada em suspensão na barra modificada (Tabela 3).

\section{Dados normativos}

A seguir serão apresentados dados normativos em atletas, visando a oferecer um referencial do nível de força em determinadas modalidades esportivas, sendo algumas apresentadas de acordo com a categoria de peso (Tabelas 4, 5).

\section{Conclusão}

É possível verificar que os testes presentes neste estudo, que avaliam a força/resistência muscular dos membros superiores do tronco, são procedimentos fáceis de aplicar, eficientes e com alto nível 
Tabela 3 - Valores em percentil para o teste de puxada em suspensão na barra modificada - modified pull-up, com base em estudos brasileiros, em pessoas saudáveis

\begin{tabular}{|c|c|c|c|c|c|c|c|c|c|c|c|}
\hline \multicolumn{12}{|c|}{ Masculino } \\
\hline \multicolumn{12}{|c|}{ Idade } \\
\hline Percentil & 7 & 8 & 9 & 10 & 11 & 12 & 13 & 14 & 15 & 16 & 17 \\
\hline 95 & 15 & 17 & 19 & 0 & 22 & 24 & 25 & 26 & 17 & 28 & 29 \\
\hline 90 & 14 & 16 & 18 & 1 & 21 & 22 & 23 & 24 & 25 & 26 & 27 \\
\hline 75 & 11 & 12 & 13 & 6 & 16 & 17 & 18 & 19 & 20 & 21 & 22 \\
\hline 50 & 8 & 9 & 9 & 10 & 11 & 12 & 13 & 14 & 15 & 16 & 17 \\
\hline 25 & 7 & 5 & 6 & 15 & 6 & 7 & 8 & 9 & 10 & 11 & 13 \\
\hline 10 & 2 & 2 & 1 & 19 & 1 & 2 & 2 & 3 & 4 & 6 & 7 \\
\hline 5 & 1 & 1 & 0 & 21 & 0 & 0 & 1 & 2 & 3 & 4 & 5 \\
\hline \multicolumn{12}{|c|}{ Feminino } \\
\hline \multicolumn{12}{|c|}{ Idade } \\
\hline Percentil & 7 & 8 & 9 & 10 & 11 & 12 & 13 & 14 & 15 & 16 & 17 \\
\hline 95 & 14 & 15 & 15 & 15 & 15 & 15 & 15 & 14 & 14 & 13 & 13 \\
\hline 90 & 13 & 13 & 13 & 13 & 13 & 13 & 13 & 13 & 13 & 12 & 12 \\
\hline 75 & 10 & 10 & 10 & 10 & 10 & 9 & 9 & 9 & 9 & 9 & 9 \\
\hline 50 & 7 & 7 & 7 & 6 & 6 & 6 & 6 & 6 & 6 & 6 & 6 \\
\hline 25 & 4 & 4 & 3 & 3 & 3 & 3 & 3 & 3 & 3 & 3 & 3 \\
\hline 10 & 0 & 0 & 0 & 0 & 0 & 0 & 0 & 0 & 0 & 0 & 0 \\
\hline 5 & 0 & 0 & 0 & 0 & 0 & 0 & 0 & 0 & 0 & 0 & 0 \\
\hline
\end{tabular}

Fonte: Guedes (6).

Tabela 4 - Dados normativos para o teste de flexão de braços/push-up

(Continua)

\begin{tabular}{|c|c|c|c|c|c|c|c|}
\hline Modalidade & Categoria & Nível & Gênero & $\mathrm{n}$ & Idade & Resultado & Ref. \\
\hline \multirow{8}{*}{$\begin{array}{l}\text { Luta olímpica } \\
\text { (Estilo livre) }\end{array}$} & $50 \mathrm{~kg}$ & \multirow{8}{*}{$\begin{array}{l}\text { Atletas iranianos } \\
\text { juniores de elite }\end{array}$} & \multirow{8}{*}{ M } & 6 & \multirow{8}{*}{$19-21$} & $69.3 \pm 7$ & \multirow{8}{*}{ (7) } \\
\hline & $55 \mathrm{~kg}$ & & & 6 & & $70.4 \pm 7$ & \\
\hline & $60 \mathrm{~kg}$ & & & 6 & & 68. \pm 5.1 & \\
\hline & $66 \mathrm{~kg}$ & & & 10 & & $69.6 \pm 6.7$ & \\
\hline & $74 \mathrm{~kg}$ & & & 11 & & $67 \pm 6.7$ & \\
\hline & $84 \mathrm{~kg}$ & & & 11 & & $64.9 \pm 7.3$ & \\
\hline & $96 \mathrm{~kg}$ & & & 10 & & $67.6 \pm 7.3$ & \\
\hline & $120 \mathrm{~kg}$ & & & 7 & & $54.2 \pm 4.6$ & \\
\hline
\end{tabular}


Tabela 4 - Dados normativos para o teste de flexão de braços/push-up

(Conclusão)

\begin{tabular}{|c|c|c|c|c|c|c|c|}
\hline Modalidade & Categoria & Nível & Gênero & $\mathrm{n}$ & Idade & Resultado & Ref. \\
\hline \multirow{8}{*}{$\begin{array}{l}\text { Luta olímpica } \\
\text { (Greco-Ro- } \\
\text { mana) }\end{array}$} & $50 \mathrm{~kg}$ & \multirow{8}{*}{$\begin{array}{l}\text { Atletas iranianos } \\
\text { juniores de elite }\end{array}$} & \multirow{8}{*}{ M } & 9 & & $59 \pm 5$ & \multirow{8}{*}{ (8) } \\
\hline & $55 \mathrm{~kg}$ & & & 9 & & $70 \pm 7$ & \\
\hline & $60 \mathrm{~kg}$ & & & 10 & & $69 \pm 9$ & \\
\hline & $66 \mathrm{~kg}$ & & & 11 & & $70 \pm 7$ & \\
\hline & $74 \mathrm{~kg}$ & & & 6 & $19-21$ & $66 \pm 7$ & \\
\hline & $84 \mathrm{~kg}$ & & & 11 & & $69 \pm 5$ & \\
\hline & $97 \mathrm{~kg}$ & & & 9 & & $70 \pm 6$ & \\
\hline & $120 \mathrm{~kg}$ & & & 6 & & $53 \pm 5$ & \\
\hline \multirow[t]{2}{*}{ Taekwondo } & $51-72 \mathrm{~kg}$ & \multirow{2}{*}{$\begin{array}{l}\text { Atletas croatas } \\
\text { de elite }\end{array}$} & \multirow[t]{2}{*}{$F$} & 6 & $21.7 \pm 4.3$ & $25.8 \pm 8.5$ & \multirow{2}{*}{ (14) } \\
\hline & $48-72 \mathrm{~kg}$ & & & 7 & $21.3 \pm 4.2$ & $23.1 \pm 7.7$ & \\
\hline \multirow{2}{*}{$\begin{array}{l}\text { Brazilian } \\
\text { jiu-jitsu }\end{array}$} & $>82 \mathrm{~kg}$ & \multirow{2}{*}{ Atletas de elite } & \multirow[b]{2}{*}{ M } & 6 & $26.3 \pm 3.6$ & $43.0 \pm 7.6$ & \multirow[b]{2}{*}{ (15) } \\
\hline & $<82 \mathrm{~kg}$ & & & 5 & $25.2 \pm 3.3$ & $36.2 \pm 8.2$ & \\
\hline Hipismo & $64.9 \pm 9.3 \mathrm{~kg}$ & Atletas colegiais & $F$ & 24 & $23.6 \pm 1.8$ & $32.1 \pm 10.6$ & (35) \\
\hline $\begin{array}{l}\text { Exército } \\
\text { brasileiro }\end{array}$ & $59.2 \pm 7.3 \mathrm{~kg}$ & Militares & $\mathrm{F}$ & 37 & $25.2 \pm 4.8$ & $22.7 \pm 4.5$ & (36) \\
\hline $\begin{array}{l}\text { Dança } \\
\text { contemporânea }\end{array}$ & $56.4 \pm 6.7$ & Profissionais & $\mathrm{F}$ & 6 & $29.6 \pm 4.4$ & $33,8 \pm 5.1$ & (37) \\
\hline Handebol & Não reportada & Atletas universitários & M & 13 & $23.7 \pm 3.1$ & $26.4 \pm 14$ & (38) \\
\hline
\end{tabular}

Fonte: Dados da pesquisa.

Tabela 5 - Dados normativos para o teste de puxada em suspensão na barra/pull-up

\begin{tabular}{|c|c|c|c|c|c|c|c|}
\hline Modalidade & Categoria & Nível & Gênero & $\mathrm{n}$ & Idade & Resultado & Ref. \\
\hline \multirow{8}{*}{$\begin{array}{l}\text { Luta olímpica } \\
\text { (Estilo livre) }\end{array}$} & $50 \mathrm{~kg}$ & \multirow{8}{*}{$\begin{array}{l}\text { Atletas juniores } \\
\text { de elite iranianos }\end{array}$} & \multirow{8}{*}{ M } & 6 & \multirow{8}{*}{$19-21$} & $40.7 \pm 9.6$ & \multirow{8}{*}{ (7) } \\
\hline & $55 \mathrm{~kg}$ & & & 6 & & $34.7 \pm 6.3$ & \\
\hline & $60 \mathrm{~kg}$ & & & 6 & & $34.6 \pm 9.3$ & \\
\hline & $66 \mathrm{~kg}$ & & & 10 & & $34.2 \pm 6.8$ & \\
\hline & $74 \mathrm{~kg}$ & & & 11 & & $32.3 \pm 6.1$ & \\
\hline & $84 \mathrm{~kg}$ & & & 11 & & $29.1 \pm 11$ & \\
\hline & $96 \mathrm{~kg}$ & & & 10 & & $21 \pm 3$ & \\
\hline & $120 \mathrm{~kg}$ & & & 7 & & $19.5 \pm 3.6$ & \\
\hline
\end{tabular}


Tabela 5 - Dados normativos para o teste de puxada em suspensão na barra/pull-up

\begin{tabular}{|c|c|c|c|c|c|c|c|}
\hline Modalidade & Categoria & Nível & Gênero & $\mathrm{n}$ & Idade & Resultado & Ref. \\
\hline \multirow{8}{*}{$\begin{array}{l}\text { Luta olímpica } \\
\text { (Greco-Romana) }\end{array}$} & $50 \mathrm{~kg}$ & \multirow{8}{*}{$\begin{array}{l}\text { Atletas juniores } \\
\text { de elite iranianos }\end{array}$} & \multirow{8}{*}{ M } & 9 & & $46 \pm 13$ & \multirow{8}{*}{ (8) } \\
\hline & $55 \mathrm{~kg}$ & & & 9 & & $35 \pm 7$ & \\
\hline & $60 \mathrm{~kg}$ & & & 10 & & $37 \pm 15$ & \\
\hline & $66 \mathrm{~kg}$ & & & 11 & & $29 \pm 11$ & \\
\hline & $74 \mathrm{~kg}$ & & & 6 & $19-21$ & $34 \pm 8$ & \\
\hline & $84 \mathrm{~kg}$ & & & 11 & & $29 \pm 12$ & \\
\hline & $97 \mathrm{~kg}$ & & & 9 & & $22 \pm 3$ & \\
\hline & $120 \mathrm{~kg}$ & & & 6 & & $17 \pm 7$ & \\
\hline $\begin{array}{l}\text { Luta olímpica } \\
\text { (Greco-Romana) }\end{array}$ & $74.75 \pm 14.80$ & $\begin{array}{l}\text { Equipe } \\
\text { polonesa }\end{array}$ & M & 61 & $18.31 \pm 91$ & $14.83 \pm 8.84$ & (13) \\
\hline $\begin{array}{l}\text { Luta olímpica } \\
\text { (Estilo livre) }\end{array}$ & $74.5 \pm 14.06$ & $\begin{array}{l}\text { Equipe } \\
\text { polonesa }\end{array}$ & M & 46 & $18.4 \pm 1.1$ & $22.14 \pm 8.26$ & (13) \\
\hline Handebol & Não reportada & $\begin{array}{l}\text { Atletas } \\
\text { universitários }\end{array}$ & M & 5 & $23.7 \pm 3.1$ & $7.2 \pm 3.4$ & (38) \\
\hline
\end{tabular}

Fonte: Dados da pesquisa.

de confiabilidade. Além disso, são ferramentas que empregam equipamento de baixo custo, que podem ser aplicadas simultaneamente e que requerem pouco treinamento do avaliador, servindo para avaliar a performance de atletas e, ao mesmo tempo, contribuir como parte do seu treinamento ou periodização. São procedimentos que podem ser aplicados em atletas de alto rendimento, em pessoas saudáveis, para a prática de atividade física, e também fazer parte de um programa de estratégias de fisioterapia. Entretanto, devemos chamar a atenção para a orientação e a padronização dos procedimentos, a fim de diminuir uma eventual interferência de fatores externos, como erros de execução, nos resultados obtidos.

Quanto aos valores obtidos, podemos inferir que os resultados variam de acordo com o gênero, a massa corporal e a modalidade esportiva. Nesse sentido, é importante que, ao selecionar o instrumento de avaliação, sejam observados alguns pontos importantes, tais como a aplicação correta das recomendações metodológicas referentes ao teste, a fim de obter qualidade nos resultados; que o teste seja pertinente à especificidade de cada modalidade ou finalidade; que o teste realmente avalie o que é proposto; e que os resultados sejam confrontados de acordo com a população.

\section{Referências}

1. Corbin CB. A multidimensional hierarchical model of physical fitness: a basis for integration and collaboration. Quest. 1991;43(3):296-306.

2. Jorgensen T, Andersen LB, Froberg K, Maeder URS, Smith LVH, Aadahl M. Position statement: testing physical condition in a population - how good are the methods? Euro J Sport Scienc. 2009;9(5):257-67.

3. Heyward V. Avaliação física e prescrição de exercício físico: técnicas avançadas. Porto Alegre: Artmed; 2004.

4. Morrow JR, Jackson AW, Disch JG, Mood DP. Medida e avaliação do desempenho humano. Porto Alegre: Artmed; 2003. 
5. Dean JA, Foster C, Thompson NN. A simplified method of assessing muscular strength. Med \& Scienc Sports \& Exer. 1987;19(2):S63.

6. Guedes D. Manual prático para avaliação em educação física. São Paulo: Manole; 2006.

7. Guedes DP. Implicações associadas ao acompanhamento do desempenho motor de crianças e adolescentes. Rev Bras Educ Fís Esp. 2007;21:37-60.

8. Eckert H, Day J. Relationship between strength and work load in push-ups. Res Q. 1967;38(3):380-3.

9. Cotten D, Marwitz B. Relationship between two flexed-arm hangs and pull-ups for college women. Res Q. 1969;40(2):415-6.

10. Baumgartner T. Modified pull-up test. Res Q. 1978;49(1):80-4.

11. Rahmani-Nia F, Mirzaei B, Nuri R. Physiological profile of elite Iranian junior Greco-Roman wrestlers. Inter J Fitness. 2007;3(2):49-54.

12. Mirzaei B, Curby DG, Rahmani-Nia F, Moghadasi M. Physiological profile of elite Iranian junior freestyle wrestlers. J Strength Cond Res. 2009;23(8):2339-44.

13. Baic M, Sertic H, Starosta W. Differences in physical fitness levels between the classical and the free style wrestlers. Kinesiology. 2007;39(2):142-9.

14. Markovic G, Misigoj-Durakovic M, Trninic S. Fitness profile of elite Croatian female taekwondo athletes. Coll Antropol. 2005;29(1):93-9.

15. Andreato LV, Franzói de Moraes SM, Esteves JVDC, Pastorio JJ, Almeida DL, Alcântara B, et al. Perfis antropométricos e funcionais de atletas de elite de jiu-jitsu de diferentes categorias. III Congresso de Ciência do Desporto - II Simpósio Internacional de Ciência do Desporto. Campinas: Unicamp; 2009.

16. Tritschler K. Medida e avaliação em educação física e esportes de Barrow e McGee. São Paulo: Manole; 2003.

17. Invergo J, Ball T, Looney M. Relationship of push-ups and absolute muscular endurance to bench press strength. J Strength \& Conditioning Res. 1991; 5(3):121.

18. Johnson BL, Nelson JK. Practical measurementes for evaluation in physical education. Minnesota: Burgess Publishing Company; 1979.
19. Mayhew J, Ball T, Arnold M, Bowen J. Push-ups as a measure of upper body strength. J Strength \& Conditioning Res. 1991;5(1):16.

20. Queiroga M. Testes e medidas para avaliação da aptidão física relacionada à saúde em adultos. Rio de Janeiro: Guanabara Koogan; 2005.

21. Rutherford O, Jones D. The role of learning and coordination in strength training. Eur J Appl Physiol Occup Physiol. 1986;55(1):100-5.

22. Pollock ML, Wilmore JH. Exercícios na saúde e na doença: avaliação e prescrição para prevenção e reabilitação. Rio de Janeiro: Medsi; 1993.

23. Dantas E. A prática da preparação física. Rio de Janeiro: Shape; 2003.

24. Marins JCB, Giannichi RS. Avaliação e prescrição de atividade física: guia prático. Rio de Janeiro: Shape; 2003.

25. Franchini E, Souza CEB, Urasaki R, Oliveira RSF, Sauressig F, Matheus L. Teste de resistência de força isométrica e dinâmica na barra com o judogi. III Congreso de la Asociación Española de Ciencias del Deporte. Madrid: INEF; 2004.

26. Silva EB, Teixeira MS, Gomes PSC. Antropometria e força muscular relativa de membros superiores. Rev Bras Fisiol Exerc. 2003;2(1):29-38.

27. Knapik J, Sharp M, Darakjy S, Jones S, Hauret K, Jones B. Temporal changes in the physical fitness of US Army recruits. Sports Med. 2006;36(7):613-34.

28. Cotten D. An analysis of the NCYFS II modified pull-up test. Res Q Exerc Sport. 1990;61(3):272-4.

29. Neto J. Caracterização dos aspectos morfológicos e de aptidão física dos escolares de Montes Claros, Minas Gerais, Brasil. Vila Real: Universidade Trásos-Montes e Alto Douro; 2009.

30. Engelman M, Morrow J Jr. Reliability and skinfold correlates for traditional and modified pull-ups in children grades 3-5. Res Q Exerc Sport. 1991;62(1):88-91.

31. Saint Romain B, Mahar MT. Norm-referenced and criterion-referenced reliability of the push-up and modified pull-up. Meas Phys Educ. Exerc Sci. 2001;5(2):67-80. 
32. Erbaugh S. Reliability of physical fitness tests administered to young children. J Perceptual and Motor Skills. 1990;71(3 Pt 2):1123.

33. Jackson A, Bruya L, Baun W, Richardson P, Weinberg $\mathrm{R}$, Caton I. Baumgartner's modified pull-up test for male and female elementary school aged children. Res Q Exerc Sport. 1982;53(2):163-4.

34. Davis K, Kang M, Boswell B, DuBose K, Altman S, Binkley H. Validity and Reliability of the Medicine Ball Throw for Kindergarten Children. J Strength Cond Res. 2008;22(6):1958-63.

35. Meyers MC, Sterling JC. Physical, hematological, and exercise response of collegiate female equestrian athletes. J Sports Med Phys Fitness. 2000;40(2):131-8.

36. La Porta MAM Jr, Fernandes J Filho, Novaes JS. Eficácia do teste de flexão e extensão de braços, corrigido pelo índice de massa corporal, na determinação da resistência muscular localizada absoluta em mulheres do exército brasileiro. 2002. [acesso 23 mar. 2011]. Disponível em: http://www. fpjournal.org.br/painel/arquivos/2162-2_Flexao_ Rev2_2002_Portugues.pdf.
37. Angioi M, Metsios GS, Twitchett E, Koutedakis Y, Wyon M. Association between selected physical fitness parameters and aesthetic competence in contemporary dancers. J Dance Med Sci. 2009; 13(4):115-23.

38. Silva AE, Almeida LR, Silva LR Jr, Rodrigues ML, Martins NS, Camargo PR, et al. Avaliação das capacidades físicas do time de handebol da Universidade Federal de Mato Grosso do Sul. Revista Digital Buenos Aires. 2010. [acesso 10 jun. 2011];14(141). Disponível em: http://www.efdeportes.com/efd 141/capacidades-fisicas-do-time-de-handebol.htm.

Recebido: 28/12/2010

Received: $12 / 28 / 2010$

Aprovado: 18/04/2011

Approved: 04/18/2011 